\title{
Monitoring and Warning Computerized System for a Semi-automatic Lift-up System of Pre-assembled Roof Floor
}

\author{
Yoshio Maruyama* and Shigeomi Nishigaki* \\ *Technical Research Institute, Hazama Corp. 515-1 Nishimukai Karima, Tsukuba, Ibaraki, \\ 305 Japan.
}

\begin{abstract}
Faced by a need to overcome work force shortage and vanishing skill replacement, a semi-automatic lift-up system of pre-assembled roof floor is developed by Hazama Corporation, which is one of general contractors in Japan. The semi-automatic lift-up system is able to automatically lift the pre-assembled roof floor up along with the progress of building frame construction. In addition, a monitor and warning computerized system is developed to monitor how the automatic lift-up system is working and the existing state of the pre-assembled roof floor, and to warn operators of the need of their intervention. As a testbed, these systems are applied to construct four stories steel structure building at the field of Technical Research Institute, Hazama Corporation. This testbed shows the constructability, operability and reliability of the systems when applying to an actual work site. This paper report the monitor and warning computerized system and its field evaluation and remarks.
\end{abstract}

\section{Introduction}

Faced by a need to overcome work force shortage and vanishing skill replacement, developing automated building construction systems have been and are being enforced in the Japanese construction industry. A semi-automatic lift-up system of preassembled roof floor is developed as shown in Figure 1 by Hazama Corporation, which is one of general contractors in Japan. The automatic lift-up system is able to automatically lift the pre-assembled roof floor up along with the progress of building frame construction. The automatic lift-up system consists of lift-up equipment, hydraulic equipment and the controllers. The lift-up equipment has several vertical posts. Each of these posts has holes at intervals of $50 \mathrm{~cm}$. As a measuring worm moving, hydraulic jacks expand, contract and move up as putting pins into and pulling ones out holes equipped with the posts, and lift up pre-assembled roof floor.

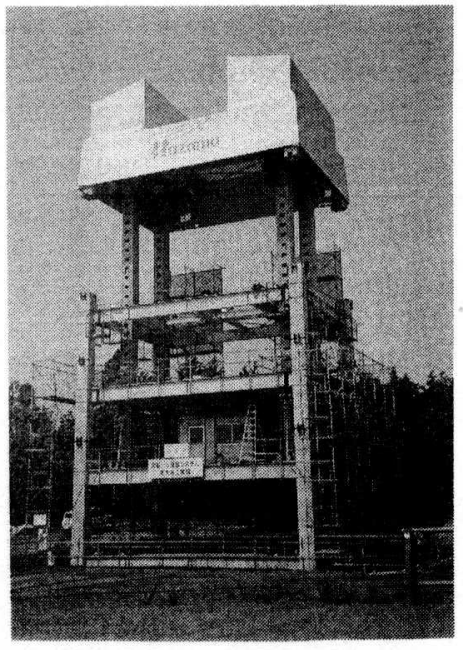

Figure 1 The lift-up system of preassembled roof floor

In this study, a monitor and warning computerized system is developed to monitor how the semi-automatic lift-up system is working and the existing state of the pre-assembled roof floor, and to warn operators of the need of their intervention. The monitor and warning system is of use to evaluate and understand the transient state of the monitored process. As 
a testbed, these systems are applied to construct four stories steel structure building at the field of Technical Research Institute, Hazama Corporation. This testbed shows the constructability, operability and reliability of the systems when applying to an actual work site.

First, this paper describes the problems and motive of this study. Second, this paper presents the monitoring and warning computerized system being developed. Third, this paper offers the field evaluation and remarks

\section{Problems and Motive}

With the semi- automatic lift-up system of pre-assembled roof floor, operators may perform the following steps.

Step 1: System start-up

- Examine system components and instrument readings to ensure that the components are readily to be put on-line.

- Once this has been done, the operators follow standard operating procedure for start-up.

Step 2: System monitoring

- Monitor its performance.

- Key process variables monitored or sensored at all points include temperature, pressure, flow rate, and so on.

- This information is continually available at computer terminals.

Step 3: Response to warnings

- In the case of system upset or equipment malfunction, warnings warn operators of the need for their intervention.

- The operators are trained to recognize warning signals and to know the appropriate warning responses.

Step 4: Shutdown of system components

Step 5: Routine maintenance. If necessary, request maintenance assistance.

Work operations here become more likely to relate to intervention, preventive maintenance, or test and replacement of components. Work operations shift from physical to increased cognitive work load in monitoring with CRT interface. At the right time and before an accident occurs, operators need to systematically keep track of hazards latent or being created in work operations. Operators here are waiting for somewhat like happenings all day along as expecting nothing may happen. Operators can not, however, pay their full attention to everything all day long. Operators become careless or absent-minded sometimes while they work. Although work schedule should be designed to avoid producing excessive fatigue, some operators will be inevitably tired from time to time. Absent-minded slips of action and bound rationality may cause an omission of attentional check. The bound rationality refers to the fact that attention can only be directed at a very small part of the total problem space at any one time. A failure to attend to change or countersigns in operations results in continuing habitual sequence of behavior.

To avoid human error and to mitigate its effect, the monitoring and warning computerized system being developed aims to provide two types of aids. One is a decision aid to easily pay direct attention to logically important aspects of the problem space and to compensate for the bound rationality. The other is a memory aid to provide a set of procedures that not only give the user a step-by-step guidance in the do's and the don'ts but also prompt operators to 
check that easily omitted steps have been completed. It is expected that the monitoring and warning computerized system enhances crew's ability through CRT interface to:

- search information,

- integrate that information, and

- take actions.

\section{The Monitoring and Warning Computerized System being Developed}

To produce a more homogeneous safety treatment of operations and to identify faults and to correct them, a decision table look-up is provided as a memory aid. The decision table look-up shows flowchart for performing operations, conditions of system start-up and shutdown, potential faults, and predetermined recovery actions or manipulations when confronted with the potential hazards. The point to be emphasized here is that key process variables and key warnings are summarized in the decision table look-up.

The key process variables represent the transient state of the semi-automatic lift-up system of pre-assembled roof floor. The key process variables are composed of signals, signs and symbols. The signals are continuous quantitative indicators of the time-space behavior of the semi-automatic lift-up system. The signals are categorized into error signals and control signals. The error signals represent the differences between the actual state and the intended or planned state in a time-space behavior. The control signals shows the transient state of behavior at a specific point in time. Signs are labeled by names of states or situations of the semi-automatic lift-up system. Signs, which are build in IF-THEN rules, are used to select or activate stored predetermined actions that control the sequence of routine operations. Symbols refer to concepts tied to functional properties and can be used for reasoning and computation by means of a suitable representation of such properties [1]. Operators can understand the existing state of the system by control signals and off-normal state by error signals. They can select and activate predetermined recovery actions by signs. Furthermore, they can reason some potential faults based on symbols, as confirming and asking for additional data. The key process variables should be monitored or sensored at all points under operations.

The key warnings mean that the warnings in questions are judged by experts to be very important for operators to identify faults and to correct them. The key warnings should be provided as a decision aid when confronted any off-normal event. Operators are trained on procedures for responding to the warning signals and to take predetermined recovery actions or manipulations under off-normal state.

Furthermore, to overcome a failure to attend any change or countersign in operations, force-gagging function is build in the monitoring and warning computerized system. The force-gagging function works to prevent the behavior from continuing until the problem has been corrected.

The structure of the inference and force-gagging mechanism based on the key process variables and key warnings are shown in Figure 2.

A great variety and volume of information on key process variables often go beyond an operator's experience and ability to digest. It is necessary to pick a set of building block of information at the right level of abstraction. In the monitoring and warning computerized system, information on key process variables is summarized into mimic diagram, symbolic information and alphanumeric information.

The mimic diagram delineates schematic overview of the semi-automated lift-up 
system and represents the physical topography of system performance in a time-space behavior, as shown in Figure 3 and Figure 4. The mimic diagram will help operators topographically investigate improper function.

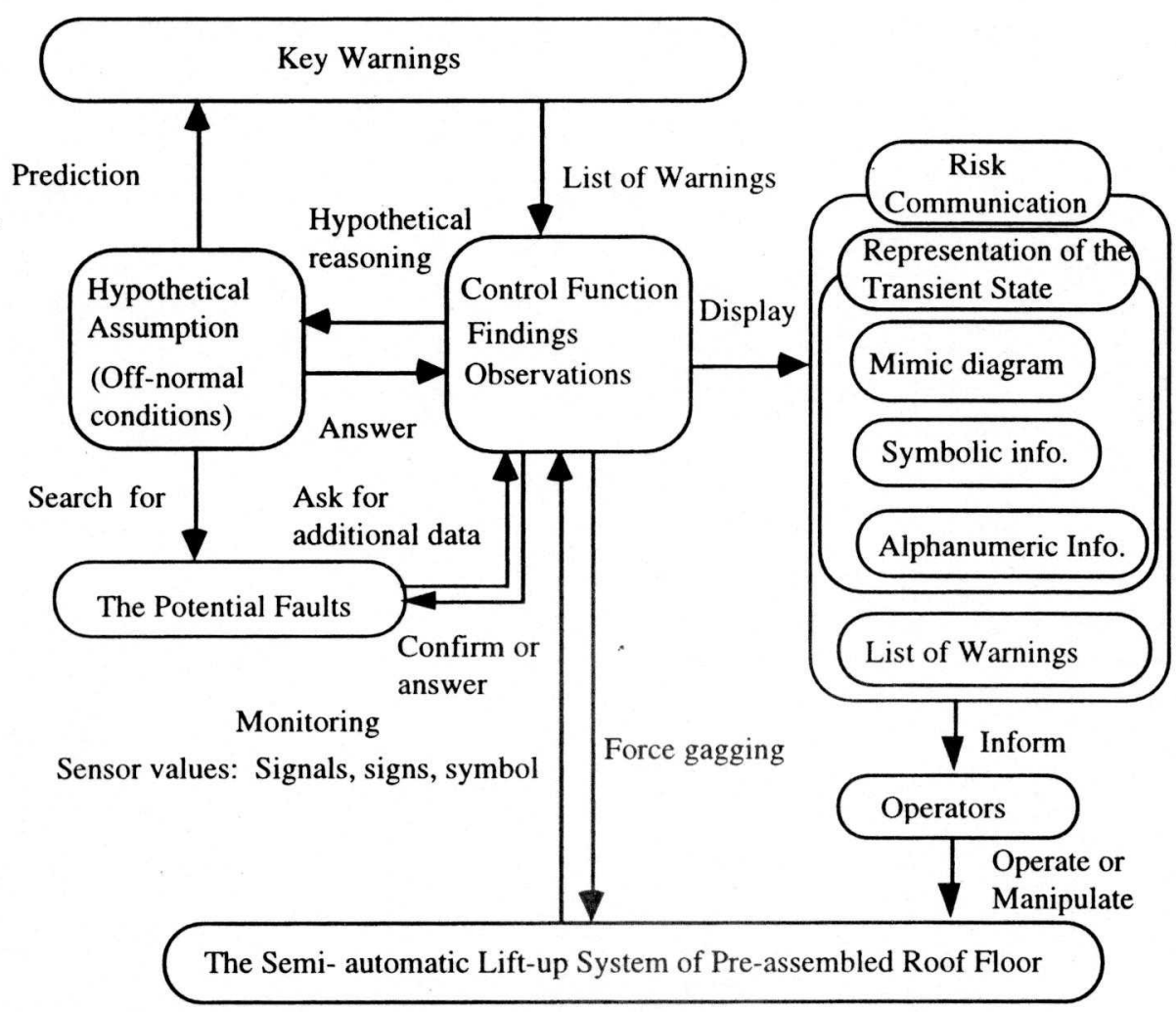

Figure 2 The Structure of the Inference and Force-gagging Mechanism

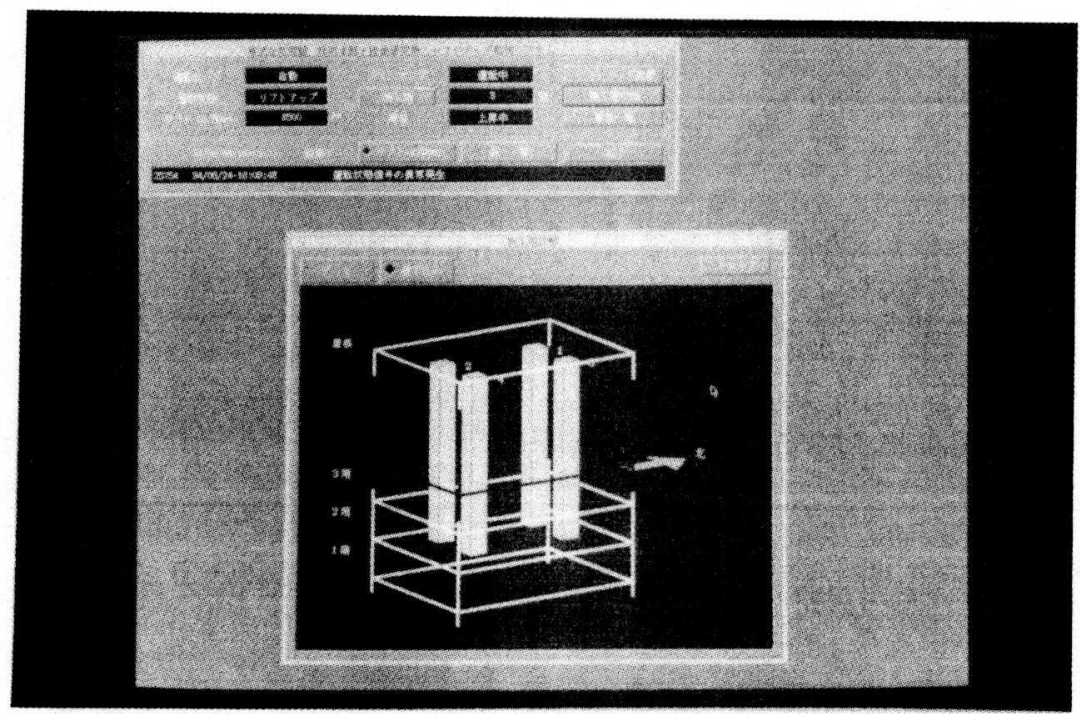

Figure 3 The mimic diagram of four stories steel structure building 


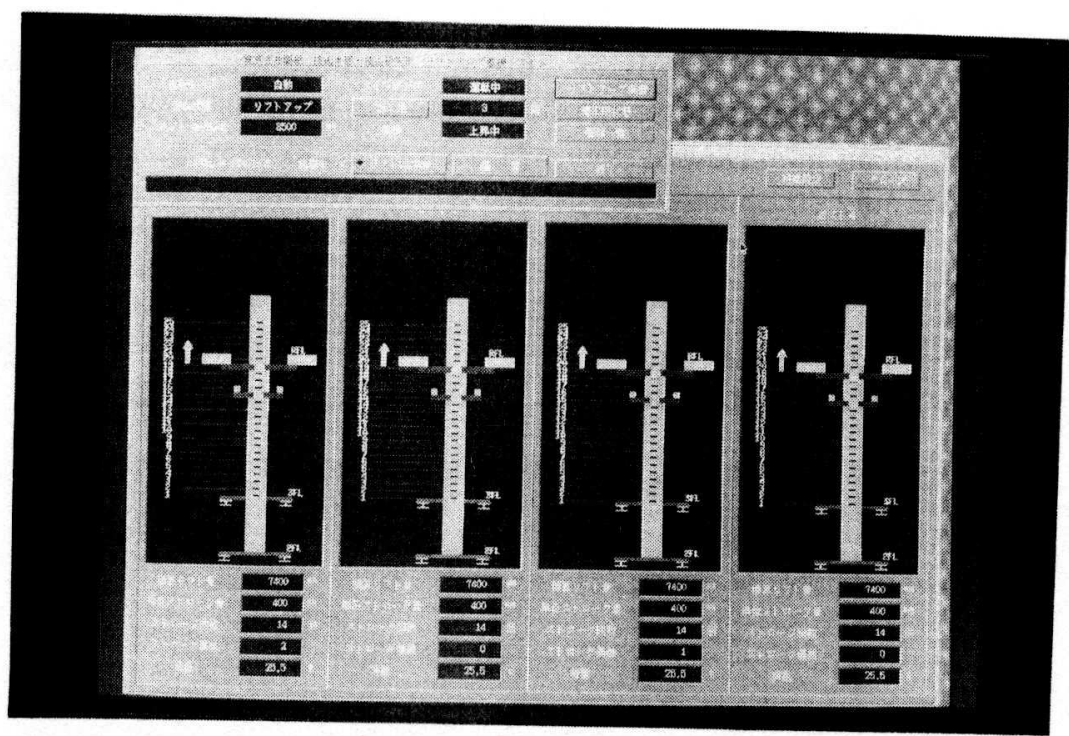

Figure 4 The mimic diagram of the semi-automated lift-up system

The symbolic information is symbolic representation of transient states of system component, for example, a direction of arrow symbolizes the direction of movement of lift-up equipment as shown in Figure 4.

The alphanumeric information display movement values of each lift-up equipment at a stroke, the number of times of lifting, and son as shown in the below of the mimic diagram in Figure 4 . These values are very important for operators to judge whether or not movement of each lift-up equipment is synchronized with others.

The key warnings are displayed as some kind of event log in the form of improper function list and unexpected event list as shown Figure 5.

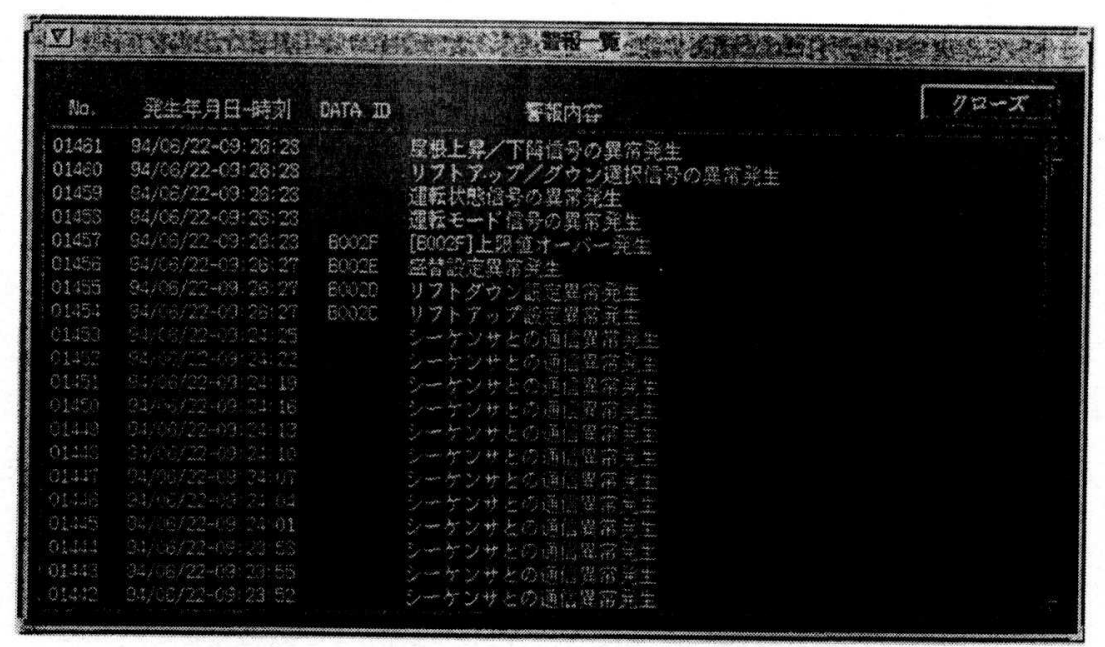

Figure 5 Key Warning List 


\section{Field Evaluation and Remarks}

As a testbed, these systems are applied to construct four stories steel structure building at the field of Technical Research Institute, Hazama Corporation. This testbed shows the constructability, operability and reliability of the systems when applying to an actual work site. During this testbed, an interview survey was conducted to hear operatorsl evaluation of the monitoring and warning computerized system. Their evaluations are summarized below.

\section{Pros}

(1) We can easily recognize the movement of lift-up equipment by the mimic diagram.

(2) Displaying arrows is very convenient and effective for us to recognize the direction of system movement.

(3) The alphanumeric information can help our judgment about the synchronization of each equipment .

\section{$\underline{\text { Cons }}$}

(1) We can not judge whether or not lift-up equipment synchronously moves by the mimic diagram.

(2) We can not recognize the movement of the pre-assembled roof floor by the mimic diagram because of its minute movement.

\section{Further Requirements}

(1) Quantitative values of the movement of the pre-assembled roof floor should be displayed on the CRT interface.

(2) In off-normal case, we need not only the warning list in sentence but also the topographic representation of the off-normal place on the CRT interface.

(3) Predetermined recovery actions or manipulations when confronted with the potential hazards should be displayed with the warning list.

To increase more situational awareness, further research and development of the monitoring and warning computerized system is required. The situational awareness refers to the crew's sensibility to the status of systems and to whether those systems are approaching their safety operating limits.

\section{References}

[1] Jems Rasmussen (1983). Skills, Rules, and Knowledge; Signals, Signs, and Symbols, and Distinctions in Human Performance Models, IEEE Transactions on Systems, Man, and Cybernetics, Vol. SMC-13, No. 3, May/June 1983.

[2] Pietro Torasso, Luca Console (1989). Diagnostic Problem Solving -combining heuristic, approximate and causal reasoning, Van Nostrand Reinhold. 